\title{
Intradable Knee Pain Caused by Foraminal Disc Herniation at the L3-4 Level
}

\author{
Dong Yeob Lee ${ }^{1 凶}$, Hoon Jae Chung ${ }^{2}$ \\ Departments of ${ }^{1}$ Neurosurgery, ${ }^{2}$ Orthopedic Surgery, Bumin Hospital Seoul, Seoul, Korea
}

A case of intractable knee pain caused by a foraminal herniated disc at the L3-4 level is reported. A 71-year-old man complained of intractable pain in the left knee despite having an unremarkable arthroscopic exploration at another hospital. Along with computed tomography and magnetic resonance imaging, coronal source images from magnetic resonance myelography revealed the foraminal herniated disc severely compressing L3 dorsal root ganglion at the L3-4 level. Surgical removal of the foraminal herniated disc at the L3-4 level improved his knee pain. The possibility of a foraminal herniated disc at the L3-4 level should, therefore, be included in the differential diagnosis of patients presenting with acute intractable knee pain.

Key Words: Lumbar vertebrae; Intervertebral disc; Radiculopathy; Knee joint

$\triangle$ Corresponding Author: Dong Yeob Lee, Department of Neurosurgery, Bumin Hospital Seoul, 389 Gonghang-daero, Gangseo-gu, Seoul 07590, Korea. Tel: +82-2-2620-0004, Fax: +82-2-2620-0167, E-mail: nsdrlee@naver.com

\section{INTRODUCTION}

Patients with foraminal/extraforaminal herniated discs can present with atypical symptoms ${ }^{2,6,10)}$. For example, patients with an extraforaminal herniated disc at the L5-S1 level may present with ankle pain ${ }^{6}$. Therefore, careless examinations of such patients may result in misdiagnosis and failed treatments.

We report a case of a foraminal herniated disc at the L3-4 level with atypical clinical presentations, i.e., acute intractable knee pain, despite unremarkable arthroscopic exploration at another hospital. Along with computed tomography (CT) and magnetic resonance imaging (MRI), coronal source images from magnetic resonance (MR) myelography were useful in diagnosing the foraminal herniated disc at the L3-4 level. The patient was treated by microdiscectomy via a lateral transmuscular route, which completely improved his knee pain.

\section{CASE REPORT}

A 71-years-old man presented with acute anterior left knee pain, which appeared suddenly 3 months ago. He did not have any history of trauma or excess activity. He had difficulty walking and sleeping due to severe left knee pain. He visited another hospital where the left knee was examined using MRI scans, and the possibility of a medial meniscus tear was suggested. The patient underwent arthroscopic exploration of the left knee, and no possible cause for pain was detected. Physical examinations showed no abnormal findings. The patient had a 2-year history of left lateral calf pain due to isthmic spondylolisthesis at the L5-S1 level and was treated conservatively by acupuncture and physical therapy. Visual analogue scale for knee pain was 10 points and lateral calf pain was 3 points (Fig. 1). A plain

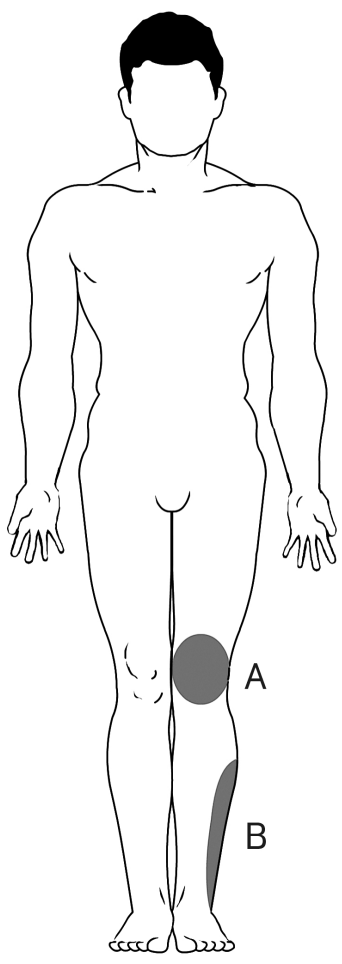

Fig. 1. Illustration of the location of the pain foci $(A$ : anterior knee pain originating from a foraminal herniated disc at the L3-4 level; B: lateral calf pain originating from isthmic spondylolisthesis at the L5-S1 level). 
radiograph showed isthmic spondylolisthesis (Meyerding grade I) at the L5-S1 level. On the lumbar MRI and CT scans, a foraminal herniated disc was detected at the left L3-4 level, along with isthmic spondylolisthesis at the L5-S1 level. Coronal source images of lumbar MR myelography showed severe compression of the L3 dorsal root ganglion by a large ruptured disc in the foraminal zones at the left L3-4 level (Fig. 2). We performed a selective left L5 nerve root block with $1 \mathrm{~mL}$ of lidocaine. After the block, his lateral calf pain markedly improved but knee pain did not improve at all. We did not perform a selective left L3 nerve root block because of the possible risk of complications, such as motor weakness after injection
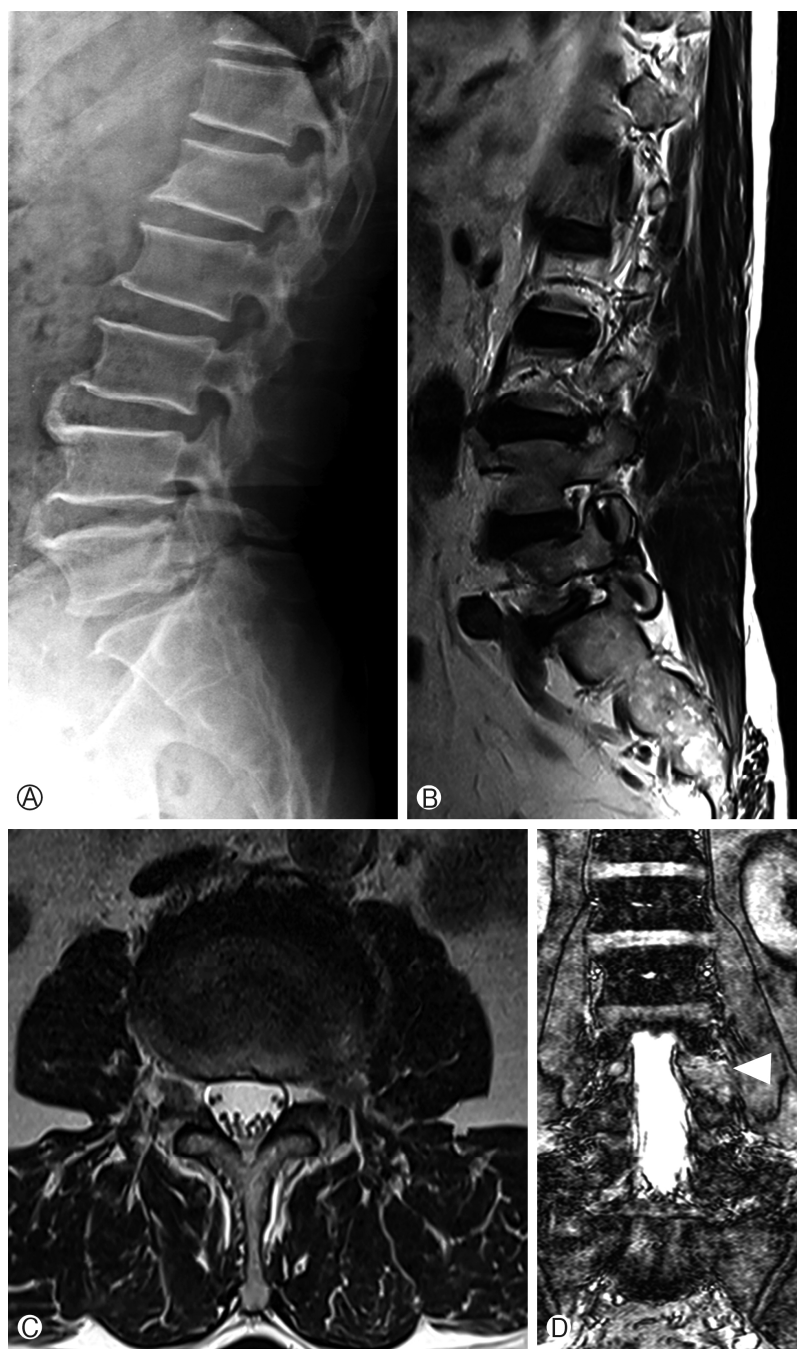

Fig. 2. Plain radiography $(\mathrm{A})$ and $\mathrm{T} 2$-weighted sagittal magnetic resonance image $(B)$ show isthmic spondylolisthesis at the L5-S1 level and a foraminal herniated disc at the left L3-4 level. T2weighted axial magnetic resonance image at the L3-4 level (C) shows a foraminal herniated disc on the left side. Coronal source image of magnetic resonance myelography clearly shows compression and obliteration of the left L3 dorsal root ganglion by a herniated disc ( $D$, arrowhead). of the dye and drug into a severely crowded foraminal zone.

Based on the patient's history and radiological findings including CT, MRI, and MR myelography, we considered the presence of an foraminal herniated disc at the L3-4 level to be the main cause of acute left knee pain. Therefore, we performed a microdiscectomy at the left L3-4 level via a modified Wiltse transmuscular approach. There were large ruptured disc fragments severely compressing the left L3 dorsal root ganglion.

Immediately after the surgery, his knee pain markedly improved. Six months after the surgery, his knee pain completely disappeared.

\section{DISCUSSION}

Lumbar disc herniations may present with pain in isolated areas in the lower extremities rather than with typical radicular pain along a sensory dermatome ${ }^{10)}$. The primary symptoms reported are pain in the thigh, calf, knee, ankle, or heel. For example, about $10 \%$ of patients with L5 or S1 lesions were reported to have asymptomatic areas between the painful foci ${ }^{2}$. Therefore, an examiner who fails to instruct the patient to completely undress and who does not perform a meticulously thorough examination can be led astray by a lack of reported symptoms ${ }^{1}$. We reported 2 atypical clinical presentations of extraforaminal herniated disc at the L5-S1 level; one patient had anterior ankle pain and the other had anterolateral thigh pain instead of typical pain on L5 sensory dermatome ${ }^{6}$. Although MRI and CT scans clearly showed extraforaminal herniated discs at the L5-S1 level, both patients were misdiagnosed and were treated on the basis of their atypical symptoms. After microdiscectomy of the extraforaminal herniated discs, their pain completely disappeared. In the present case, the patient complained of severe anterior knee pain. His condition was initially misdiagnosed as a knee joint problem. Consequently, arthroscopic exploration of the knee joint was performed, and this did not improve his pain at all.

In cases where MRI findings do not coincide with the patient's symptoms, a thorough neurological examination is extremely important $^{7}$. Multi-access assessments such as digital infrared thermographic imaging, MRI, MR myelography, and/or selective nerve root block, are warranted ${ }^{7}$. We concluded that a foraminal herniated disc at the L3-4 level was causing the acute knee pain. Firstly, the patient did not have any history of trauma or excess activity that might have caused acute knee joint pain. Secondly, arthroscopic exploration of the knee joint neither showed any pathological lesions nor did it improve the knee pain. Thirdly, a selective diagnostic L5 nerve root block markedly improved the patient's lateral calf pain but did not improve his knee pain. Lastly, coronal source images of MR myelography demonstrated a large ruptured disc compressing the L3 dorsal root ganglion at the foraminal zone on 
the left L3-4 level. Since anterior knee area is included in the L3 sensory dermatome, a herniated disc compressing the L3 root was considered to be the cause of the isolated knee pain. After a microdiscectomy at the L3-4 level, the knee pain improved drastically.

Diagnostic nerve root blocks safely and accurately discern the presence or absence of lumbar radiculopathy ${ }^{6,7,9)}$. Sasso et al. reported that $91 \%$ of the patients with a positive selective nerve root block had good surgical outcomes, whereas only $60 \%$ of patients with a negative selective nerve root block had good surgical outcomes'). In our previous report on an extraforaminal herniated disc at the L5-S1 level presenting with atypical pain, we performed a selective nerve root block with $1 \mathrm{~mL}$ of lidocaine to confirm the extraforaminal herniated disc as a cause of atypical pain ${ }^{6}$. After the block, the pain improved transiently and disappeared completely after a subsequent microdiscectomy. In the present case, it would have been beneficial to perform a selective left L3 nerve root block. However, we decided against it to avoid the possible risk of motor weakness after injection of the dye and drug into a severely crowded foraminal zone. Instead, we performed a selective left L5 nerve root block to rule out isthmic spondylolisthesis at the L5-S1 level as a cause of knee pain.

Recent advances and refinements in diagnostic imaging modalities have markedly improved preoperative diagnosis of pathological lesions in the foraminal/extraforaminal zone ${ }^{4}$. However, correct diagnosis of pathologic lesions located in the foraminal zone is sometimes difficult even with $\mathrm{CT}$ and/or MRI scans. The pathological lesions in the foraminal zone have frequently been considered the cause of persistent symptoms despite surgery ${ }^{1,3}$. In the present study, CT and MRI scans revealed a foraminal herniated disc at the L3-4 level, which was initially not considered as the cause of acute knee pain. However, coronal source images of MR myelography clearly showed severe compression of the L3 dorsal root ganglion by a herniated disc in the foraminal zones. Coronal source images of MR myelography are the source images used to extract conventional MR myelography images. These images can clearly define the anatomical structures of the foraminal/extraforaminal areas of the entire lumbar spine, including the pedicle, ganglion, and foraminal/extraforaminal disc. These images are, therefore, helpful in diagnosing pathological lesions in the foraminal/ext- raforaminal zones when combined with CT and MRI scans ${ }^{4,8)}$. Dorsal root ganglion swelling and running course abnormalities are 2 findings useful in diagnosing root compression at the foramen/extraforamen ${ }^{5}$.

\section{CONCLUSION}

We report a case of acute intractable knee pain caused by foraminal herniated disc at the L3-4 level. The possibility of a foraminal herniated disc at the L3-4 level should be included in the differential diagnosis of patients presenting with acute intractable knee pain.

\section{REFERENCES}

1. Burton CV, Kirkaldy-Willis WH, Young-Hing K, Heithoff KB: Causes of failure of surgery on the lumbar spine. Clin Orthop Rel Res 157:191-199, 1981

2. Friis ML, Gulliksen CG, Rasnussen P, Husby J: Pain and spinal root compression. Acta Neurochir 39:241, 1977

3. Jang JS, An SH, Lee SH: Clinical analysis of extraforaminal entrapment of L5 in the lumbosacral spine. J Korean Neurosurg Soc 36:383-387, 2004

4. Kim JU, Lee SH, Lee DY: Extraforaminal lumbar synovial cyst causing sudden foot drop. Neurol Med Chir (Tokyo) 48:578-581, 2008

5. Kim SB, Jang JS, Lee SH: Morphologic changes of L5 root at coronal source images of MR myelography in cases of foraminal or extraforaminal compression. J Korean Neurosurg Soc 46:1115,2009

6. Kim SE, Lee SH, lee DY, Lee HS: Atypical clinical presentation of lumbosacral extraforaminal disc herniation- two case reports. Kor J Spine 3:171-174, 2006

7. Lee DY, Kim HS, Lee SH: Multi-access for the diagnosis of missed upper lumbar disc herniation. J Korean Neurosurg Soc 38:144146,2005

8. Lee DY, Lee SH, Lee HS: Unilateral isthmus resection for elderly foraminal stenosis. J Korean Neurosurg Soc 41:207-209, 2007

9. Sasso RC, Macadaeg K, Nordmann D, Smith M: Selective nerve root injection can predict surgical outcome for lumbar and cervical radiculopathy: Comparison to magnetic resonance imaging. J Spinal Disord Tech 18:471-478, 2005

10. Wisneski RJ, Garfin SR, Rothman RH, Lutz GE: Lumbar disc disease, in Herkowitz HN, Garfin SR, Balderston RA, Eismont FJ, Bell GR, Wiesel SW (eds): The Spine. Philadelphia: WB Saunders, 1999, pp613-679 